Ebisu Ebisu

Études japonaises Études japonaises

$58 \mid 2021$

2011-2021 : crises, ruptures et nouvelles dynamiques.

Dix ans après la triple catastrophe du 11 mars

\title{
RIBAULT Thierry, Contre la résilience de Fukushima et ailleurs
}

Paris, L'échappée, 2021, 365 p.

\section{Anne Gonon}

\section{(2) OpenEdition}

\section{Journals}

Édition électronique

URL : https://journals.openedition.org/ebisu/6233

DOI : 10.4000/ebisu.6233

ISSN : 2189-1893

Éditeur

Institut français de recherche sur le Japon à la Maison franco-japonaise (UMIFRE 19 MEAE-CNRS)

Édition imprimée

Date de publication : 1 novembre 2021

Pagination : 361-365

ISSN : $1340-3656$

Référence électronique

Anne Gonon, « RIBAULt Thierry, Contre la résilience de Fukushima et ailleurs », Ebisu [En ligne], 58 | 2021, mis en ligne le 01 novembre 2021, consulté le 14 avril 2022. URL : http://journals.openedition.org/ ebisu/6233; DOI : https://doi.org/10.4000/ebisu.6233 


\section{Comptes rendus}

Une catastrophe n'est pas seulement un événement qui s'inscrit dans une temporalité courte et dans un espace circonscrit, mais, prise dans le travail de reconstruction et un ensemble de discours politiques, scientifiques et sociaux, elle devient un objet pour recomposer le monde.

C'est de cet objet que traite l'ouvrage de Thierry Ribault, dont la complexité traduit bien l'opacité de ces opérations de reconstruction qui, depuis dix ans, font de la triple catastrophe du Japon de l'Est, une expérimentation de la vie dans un espace contaminé par des radiations à la suite d'un accident nucléaire. $\mathrm{Au}$ cour de l'abondante production de travaux et d'œuvres artistiques et littéraires parus en français, en japonais et en anglais, l'ouvrage de Thierry Ribault occupe une place particulière par la radicalité de sa démarche. Certes, il traite de cette catastrophe, mais - ainsi que le soustitre nous l'indique - pas seulement de Fukushima mais aussi d'ailleurs, d'un ailleurs géographique, puisqu'il y est aussi question de Tchernobyl, de Three Miles Island et d'un ailleurs social telle la pandémie dans laquelle nous vivons depuis plus d'un an. Thierry Ribault, chercheur en sciences sociales rattaché au CNRS, 
travaille depuis dix ans sur la catastrophe survenue le 11 mars 2011 dans la centrale nucléaire de Fukushima, période au cours de laquelle il a arpenté la région sinistrée, rencontré et interrogé des chercheurs, des personnes touchées par la catastrophe, des décideurs japonais et étrangers. L'ouvrage expose une réflexion à la fois réceptive aux transformations de la gestion de cette catastrophe au cours de ces dix ans écoulés, et ancrée dans un cadre théorique solide, celui de la théorie critique de l'école de Francfort ${ }^{1}$. La rigueur méthodologique s'allie à une utilisation de sources variées, fines et précises pour dresser une critique de la violence de notre société, qui a rendu possible une telle catastrophe nucléaire. Pour Thierry Ribault, il semble que la compréhension de ce que la catastrophe fait à notre vie doive être placée au cœur d'une critique de la modernité, et surtout des mécanismes invisibles qui la transforment en un processus insaisissable, et qui favorisent la déshumanisation.

$\mathrm{Si}$, dans les premiers temps qui ont suivi la survenue de la catastrophe, des voix critiquant la modernité et sa course technologique ont tenté de se faire entendre, elles sont devenues inaudibles, voire ont disparu au profit d'une approche pragmatique de la situation, d'un accompagnement de ce que le gouvernement japonais, mais aussi les individus cherchaient à faire - comment reconstruire, comment retrouver une vie normale?

Ce sont ces comportements qu'interroge Thierry Ribault, à partir de ce qui est la notion-clé qui circule entre toutes les parties concernées: la résilience. Plus encore, l'auteur saisit la résilience en ce qu'elle s'articule à d'autres notions également fondamentales telles que la production de l'ignorance, le consentement et le savoir citoyen, pour "faire du malheur un mérite".

L'ouvrage est construit en huit parties aux titres aussi percutants qu'angoissants: "Ce carnage au bruit sinistre "; "Le despotisme de la résilience "; "La production de l'ignorance »; "La falsification du monde "; « La perte, c'est la porte? "; "Gouverner par la peur de la peur "; "Les galères citoyennes "; "Le malheur n'est pas un mérite».

La résilience (partie 2) est la notion centrale de toutes les réflexions et actions menées après la catastrophe nucléaire comme de toute autre forme de désastre. L'auteur mentionne les nombreux domaines - biologique, psychologique, social-, où cette notion est mise en avant depuis quelques années pour parler des individus confrontés à des bouleversements sociaux, environnementaux, 
sanitaires. Elle est considérée comme le moyen d'affronter ce qui advient et de se préparer à ce qui pourrait advenir. Elle s'est peu à peu imposée dans le champ des politiques de gestion des crises, comme une aptitude naturelle dont il suffirait de prendre conscience et de mieux connaitre afin que l'être humain en fasse une ressource constructive: même les victimes de PTSD (traduit en français par «le trouble de stress post-traumatique») devraient ainsi pouvoir rebondir.

Mieux encore, dans le cas d'une exposition à la radioactivité, les autorités demandent que les personnes exposées apprennent à vivre " malgré l'adversité et la souffrance» (p. 55), permettant ainsi de ne pas remettre en cause le programme nucléaire luimême: des programmes, appelés dialogues, soutenus par les instances internationales, sont mis en place et proposent aux personnes vivant dans les zones contaminées les moyens de gérer leur santé. Thierry Ribault dénonce les ressorts de ce discours ou "de cette formule magique de substitution" (p. 61) dont il veut déconstruire le caractère idéologique et déshumanisant.

Cette résilience est rendue possible par un ensemble de mécanismes qui produisent de l'ignorance (partie 3). Lauteur évoque, à la suite des travaux de Robert Proctor sur le tabac ${ }^{2}$, la science " non faite " - la discussion sur les faibles doses qui provoque une controverse alors que certains travaux démontrent la dangerosité de l'exposition aux radiations, mais aussi les études incomplètes qui omettent de prendre en compte des symptômes autres que le cancer de la thyroïde ou qui utilisent des échantillons alimentaires discrétionnaires.

Le terme de résilience permet ainsi de mettre en place une économie du consentement (partie 4), dans laquelle la production d'ignorance conduit à afficher, sur la base de ce qui est considéré comme connu, des mesures et des seuils d'acceptabilité sociale. Ainsi est construit un monde "faux" qui ne permet pas aux individus de connaitre exactement la mesure des dangers ni d'engager des actions pour se protéger, mais les amène à accepter et cogérer les catastrophes dont ils sont les victimes. Selon Thierry Ribault, cette fausseté du monde dans lequel les victimes doivent vivre fait d'elles les véritables personnes déplacées, car vivant dans un lieu impropre à la vie, alors que nous désignons habituellement comme personnes déplacées celles qui se sont réfugiées dans d'autres départements. Il écrit : «les déplacés sont projetés dans un monde faux qui ne leur appartient pas et dans lequel leur nature ne peut se révéler» (p. 152). 
Vivre dans un monde faux, c'est faire de la perte (partie 5) une ressource pour rebondir: la nuisance radioactive n'est pas combattue, mais devient l'environnement d'une nouvelle vie, d'une nouvelle forme de vie. Il suffira d'accepter de vivre en prenant des précautions. Voilà ce qui fonde les politiques de la résilience dans lesquelles les individus sont amenés à se former et à devenir des citoyens actifs. Les autorités parlent d'empowerment. Ce processus d'adaptation, encouragé par les discours sur la résilience, devient une technologie du consentement, porteuse d'espoir. Ce processus n'est pas possible sans une manipulation des affects, et notamment de la peur (partie 6). L'insistance sur la résilience permet que toutes les réactions les plus normales face à un danger aussi pernicieux que le danger de la contamination nucléaire soient traitées comme des marques de faiblesse, d'incapacité à s'adapter. Il suffit de se rappeler la déclaration du professeur Yamashita, affirmant que le sourire éloigne le risque de contamination. Thierry Ribault démonte les mécanismes qui tentent de promouvoir un être humain adapté à une société qui veut en disposer à sa guise. N'existe-t-il pas des citoyens conscients de ces mécanismes idéologiques (partie 7)? On a beaucoup parlé de ces personnes sinistrées qui ont cherché à se renseigner sur les effets de la radioactivité et ont organisé leurs propres lieux et instruments de mesure. Ne peut-on pas voir dans de telles initiatives dites de sciences citoyennes une sortie possible de cet enfermement idéologique? Ici, l'auteur montre à la fois sa position en déclarant que l'activisme du citoyen n'aide pas à " accéder à une pleine et réelle conscience de son impuissance qui lui permettrait de rester, malgré tout, maître de lui-même " (p. 280).

En conclusion, l'on peut dire que le style est mordant, parfois ironique, à la hauteur de l'exigence d'authenticité qui guide l'auteur : il n'est pas question d'esthétiser le désastre, mais de traiter de la mort, de notre dépossession de la capacité à décider (ou non) de notre vie. La lecture de l'ouvrage de Thierry Ribault est nécessaire, tant le terme de résilience résonne actuellement comme un mot magique pour penser la sortie de la catastrophe du 11 mars. En démystifiant et déconstruisant cette notion, Thierry Ribault se place en complément des - trop rares apports critiques, si l'on excepte ceux de Magali Reggheza-Zitt en géographie ou d'Eva Illouz en sociologie. Louvrage de Thierry Ribault est salutaire en ce qu'il se refuse à essentialiser le comportement des Japonais et met à nu les mécanismes de cette 
dépossession, produite par la modernité. L'auteur nous laisse la difficile tâche de transformer cette connaissance en action.

Anne Gonon

Professeure à

l'université Dōshisha

1. En replaçant la catastrophe du 11 mars 2011 dans le cadre du processus de modernisation, pour en comprendre l'origine et l'idéologie qui la porte, tout en donnant des outils pour s'émanciper de ce processus idéologique, on peut dire que la recherche de Thierry Ribault s'inscrit dans la tradition de la théorie critique l'École de Francfort et notamment dans la démarche de Horkheimer.

2. Robert N. Proctor, historien des sciences est l'auteur de Golden Holocaust, la conspiration des industries du tabac, Paris, Éditions des Équateurs, 2014..
Ce livre est le résultat d'un projet de recherche collectif mené en 2016 sur l'impact économique et financier des catastrophes naturelles de grande ampleur (mega disasters) au Japon. Il comprend six chapitres. Le premier chapitre (p. 1-13), rédigé par Kamesaka Akiko et Franz Waldenberger, sert d'introduction générale en présentant la problématique globale du livre, autour de laquelle se coordonnent les cinq autres chapitres. Les auteurs partent du constat selon lequel les pertes et les dégâts causés par des catastrophes de grande ampleur ont significativement augmenté depuis les dernières décennies. Par exemple, le Japon, qui est l'un des pays les plus exposés aux séismes, recense, entre 1990 et 2017, $40 \%$ des pertes et des dégâts liés à un tremblement de terre au niveau mondial. À la force destructrice de ces phénomènes s'ajoute la forte concentration des actifs dans les zones à risque expliquant l'ampleur des conséquences 